\title{
Tangence
}

\section{Benoîte Groult et Paul Guimard : l'écriture en deux temps et quelques mouvements}

\section{Lucie Joubert}

Numéro 62, avril 2000

Parentèle

URI : https://id.erudit.org/iderudit/008177ar

DOI : https://doi.org/10.7202/008177ar

Aller au sommaire du numéro

Éditeur(s)

Presses de l'Université du Québec

ISSN

0226-9554 (imprimé)

1710-0305 (numérique)

Découvrir la revue

Citer cet article

Joubert, L. (2000). Benoîte Groult et Paul Guimard : l'écriture en deux temps et quelques mouvements. Tangence, (62), 123-138.

https://doi.org/10.7202/008177ar d'utilisation que vous pouvez consulter en ligne.

https://apropos.erudit.org/fr/usagers/politique-dutilisation/ 


\section{Benoîte Groult et Paul Guimard : I'écriture en deux temps et quelques mouvements}

\section{Lucie Joubert, Université Queen's}

On connaît Benoîte Groult surtout pour son implication féministe, en particulier son essai Ainsi soit-elle et pour les romans qu'elle a écrits en collaboration avec sa sour Flora, romans longtemps qualifiés d'ouvrages de dames, boudés dans les années 1970 par la critique institutionnelle mais courus par un public féminin qui s'évadait avec plaisir dans des univers très proches de ce quotidien qu'il cherchait alors pourtant à fuir. Au Québec par contre, on a peut-être un peu moins lu Paul Guimard, son époux depuis quarante ans, auteur de fictions savoureuses toutes empreintes d'ironie. On ignore totalement, sans doute, combien leurs écritures se rejoignent sans jamais cesser de se contester, se "chicanent" même pour mieux fournir à des questions universelles des réponses divergentes mais jamais dissonantes.

À même leur vie commune, les deux écrivains ont en effet tissé des œuvres qui s'élaborent autour de paramètres étrangement similaires qui confèrent à l'ensemble, non pas une impression de redite ou de déjà $l u$, mais plutôt la sensation d'une complicité qui s'étend au-delà de la vie conjugale pour atteindre à une communion des sens. Des sens, dis-je bien, car l'étroite parenté qui lie les deux écritures relève beaucoup plus d'une volonté de saisir - de ressentir - les infinies nuances du monde (ses odeurs, ses bruits, ses saveurs, ses couleurs, ses inflexions et ses êtres, surtout) que d'en expliquer le fonctionnement. Si bien sûr les consciences se rejoignent, exprimant une pensée parallèle, une façon convergente, littéralement, de prendre position devant l'univers, ce n'est jamais au détriment de cette urgence de vivre qui fait le bonheur des lecteurs.

Groult et Guimard apposent sur leurs œuvres respectives trois sceaux distincts qui scellent leur parenté : la mer, le temps ${ }^{1}$, l'ironie.

1 Cette récurrence tourne presque à l'obsession si on en juge par les titres des œuvres publiées ou les thèmes abordés par cette prolifique "famille": chez Paul Guimard, dans Les choses de la vie, un homme sur le point de mourir 
Récurrentes dans tous leurs romans (avec plus ou moins d'importance selon la trame romanesque), ces composantes marquent leurs écritures tant sur le plan thématique que narratologique et rhétorique. Deux textes, en particulier, confirment la jonction des styles: La part des choses, pour Groult, et Le mawvais temps, pour Guimard, publiées respectivement en 1972 et $1976^{2}$ alors que les auteurs avaient 52 et 55 ans. Romans de la maturité, donc, que ces œuvres qui adoptent la mer comme toile de fond et comme lieu privilégié du bilan et de la redécouverte de soi. Les deux ouvres s'articulent, chacune de son côté, autour d'une quête personnelle, une traversée des âges en fait, pendant laquelle Marion et Monsieur Robert, personnages centraux respectifs des romans, vont se mesurer à eux-mêmes. La similarité des projets va cependant mettre en évidence la non-neutralité des univers ${ }^{3}$ des deux écrivains marqués (malgré eux?) par leur appartenance sexuelle: différence de perception du temps, selon que l'on est homme ou femme, divorce des procédés narratologiques, variation des techniques de l'ironie.

\section{La mer comme lieu commun}

"L'espace forme l'antidote du souci et la sagesse populaire a raison de penser que les voyages calment les peines. Encore fautil que l'âme consente à voyager avec le corps ", écrit Burgelin. Dans les deux romans qui nous intéressent ici, un voyage en bateau agira comme révélateur d'un changement profond chez le personnage principal. Dans La part des choses, Marion, 45 ans,

revoit défiler toute sa vie; dans Rue du Havre, quelques minutes séparent deux destins que la vie se chargera de réunir; Un concours de circonstances raconte la même séquence temporelle, vue sous différents angles. Chez les sœurs Groult, Journal à quatre mains, par la narration d'une jeunesse volée à toute une génération, se révèle plus une chronique de l'Occupation qu'un roman; Il était deux fois relate la réunion de deux amies d'enfance qui découvrent que le fils de l'une est l'amoureux de la fille de l'autre. Chez Benoîte, ensuite, Les trois quarts du temps constitue le bilan d'une vie de femme, Les vaisseaux du cour nous emporte dans la liaison extra-conjugale passionnée que George (une femme) entretient toute sa vie durant avec Gauvain. Chez Flora, les titres mêmes se passent de commentaires: Un seul ennui, Les jours raccourcissent, Le temps s'en va, Madame, Le passé infini, Une vie n'est pas assez.

2 Dorénavant, les références seront indiquées comme suit: PC et $M T$.

3 J'emprunte cette formulation à Louise Dupré.

4 Pierre Burgelin, L'bomme et le temps, Paris, Aubier, Éditions Montaigne, 1945, p. 20 
part en croisière autour du monde avec Yves son mari et deux couples d'amis. Le périple lui permettra, espère-t-elle, de faire le point sur sa vie mais surtout de se débarrasser du fantôme de Yang, la jeune maîtresse d'Yves qui vient de se suicider. Dans Le mauvais temps, Robert, dans la jeune cinquantaine, part en voyage, seul sur son bateau, pour se redéfinir à l'aube d'une nouvelle relation amoureuse.

Pour Marion, femme de racines, le départ, si excitant soit-il (qui ne rêve pas d'une croisière de ce genre?), ne suscite pas l'enthousiasme escompté :

Quand on peut s'asseoir au cour de son jardin avec la sensation béate d'être au centre du monde, quand on sait que par vent de sud-est on entendra la bouée de Merrien et qu'on l'entend et qu'on se dit avec une satisfaction toujours nouvelle: "Tiens, la bouée sifflante de Merrien! Le vent est à suet..."; quand on s'obstine à trouver que le fschfsch des vagues sur sa plage est plus émouvant que les autres fschfsch, quand enfin on éprouve le besoin de retrouver l'odeur de son pays, comme un médicament, chaque fois que l'on est malheureux, pourquoi courir ailleurs? ( $P C$, p. 7$)$

Réticente devant la perspective d'un tête-à-tête prolongé avec son mari, après la tempête conjugale qu'ils viennent tous deux d'essuyer, Marion, femme lucide et rationnelle, redoute aussi de "tomber dans le panneau des lointains voyages, alibi ou miroir aux alouettes de tant de médiocres" ( $P C$, p. 9). Cependant, "ce qu'elle voulait, c'était ce qui lui arrivait précisément: que des circonstances indépendantes de sa volonté l'amènent à partir tout en lui permettant d'exprimer des réserves" ( $P C$, p. 9): un appareillage à son corps à moitié défendant, en somme, qui fait naître en elle des sentiments contradictoires.

Dans Le mauvais temps au contraire, Robert largue les amarres de son voilier sur un coup de tête, en un geste à la fois consenti mais non prémédité. Il vient de recevoir une lettre d'Anne, sa maîtresse, lui demandant de venir la rejoindre en Hollande : " $\mathrm{A}$ dix heures ce matin, ma secrétaire m'a retenu une place sur le vol Paris-Amsterdam. À onze heures, je lui ai demandé d'annuler la réservation. Je sais ce que je vais faire. J'aurais dû y penser plus tôt" ( $M T$, p. 74). Dans un mouvement dont la finalité ne transparaît pas sur-le-champ pour le lecteur, le personnage tourne le dos à la femme aimée pour affronter la mer en Bretagne. C'est en pleine tempête qu'il réglera ses comptes avec lui-même. 
Fuite en avant, les deux navigations ne poursuivront pas les mêmes objectifs mais atteindront le même but, par des voies différentes: alors que Marion cherche surtout à se réconcilier avec la vie et avec son mari ( $P C$, p. 47), Robert Duchesne tente de concilier les deux parties de lui-même qui s'affrontent: Bob, le jeune homme et Monsieur Robert, l'homme d'âge mûr. Les procédés narratologiques privilégiés par les deux auteurs participeront alors de cette différence. Chez Groult, en effet, la narration extérieure cède par moment la place à une écriture du je - les cahiers Gallia — sorte de journal de bord de la voyageuse dans lequel elle "ne [dira] pas toute la vérité. Peut-être rien que la vérité : c'est déjà beaucoup" ( $P C$, p. 47). Ces pages créent une rupture du rythme de la narration en ce qu'elles permettent, dans le changement de points de vue, de refaire avec Marion le parcours de ses deux dernières années de vie conjugale ${ }^{5}$. Le journal déplace en outre la perspective afin de donner à lire une autre strate du personnage: la Marion racontée par la narratrice usurpe la parole pour donner $s a$ version du voyage et de la tragédie intime qui a marqué sa vie de couple. Ainsi se trouve créé, à même l'alternance narratologique, un mouvement de va-et-vient qui n'est pas sans rappeler les hésitations du personnage même et le roulis du bateau sur lequel elle espère trouver réponse à ses questions. Deux "voyages", en somme, se superposent. D'un côté, le voyage intérieur, itinéraire affectif, houleux et douloureux parce qu'il oblige Marion à remuer de vieilles eaux dans cette volonté de noyer le passé et de changer de cap; de l'autre, l'itinéraire réel, la croisière de luxe, décevante pour Marion à première vue, car

ce tour du monde, ce rêve énorme qui habite tant de cours [semble] se réduire à cette balade pittoresque qui ne changeait rien de fondamental ni en elle ni en eux. [...] Quand une ville leur plaisait, ils l'accrochaient au passage et la maintenaient au bout de leur ancre, le temps de descendre. Puis ils la relâchaient et la ville s'écartait doucement pour laisser la place à une autre (PC, p. 169).

En fait, le cahier Gallia permet à Marion de "vivre ce voyage au lieu de le regarder" ( $P C$, p. 170) et d'échapper à l'univers aseptisé, codifié, du Moana pour partir à l'aventure, la vraie, si l'on peut dire, à la recherche de la "paix du cœur" ( $P C$, p. 52).

5 À ces deux voix s'en greffe une troisième, celle de l'ironiste, sur laquelle je reviendrai plus loin. 
Si le cahier Gallia, sorte de miroir de l'âme de l'héroïne, nous introduit épisodiquement dans les pensées secrètes de Marion, laissant à la narratrice extérieure le soin de brosser le tableau général des incidents de parcours vécus par tous les personnages, la narration homodiégétique du Mauvais temps va au contraire resserrer le point de vue autour d'un nous qui, lui, se dédouble, sorte de je à deux faces, donnant à ses deux entités fondues en une le loisir de s'observer et de s'entre-commenter:

Monsieur Robert bascule son amas d'organes pondéreux pour dégager les jambes des draps, poser les pieds sur le sol en redressant le torse et il reste assis un instant au seuil de cette journée de plus qu'il faudra parcourir. Puis il met debout ses kilos harassés par le mauvais sommeil et accomplit avec incertitude les pas qui le séparent du lavabo dans lequel il se vidange jusqu'à la lie. C'est l'heure que je déteste entre toutes. Chaque matin je cherche mon visage dans la glace et je ne bois que le reflet d'un autre. Nous avons un vague air de famille, cela est certain, cet important personnage pourrait être mon oncle, mais où suis-je? Le miroir déformant ne me renvoie que ma caricature. Bon gré, mal gré, c'est Monsieur Robert qu'il me faut contempler (MT, p. 16).

Commence alors un astucieux monologue à deux voix: dans la première partie, donc, on n'entendra que le jeune Robert se moquer de cet Autre qu'il ne veut à aucun prix reconnaître comme celui qu'il est devenu et à qui, surtout, il ne veut pas céder la place. La deuxième partie, la fuite en avant dont il est fait état plus haut, c'est-à-dire ce qui devait être une agréable excursion en bateau pour faire le point, permettra à Monsieur Robert, avec sa longue expérience de marin, de sauver la vie de Bob, le jeune, en grand danger sur une mer démontée. Danger qu'il sous-estime superbement, convaincu qu'il est de pouvoir affronter tous les temps: "Depuis longtemps je rêve d'entraîner l'Autre dans des circonstances et dans un lieu qui le rendent subalterne: nous y voici" (MT, p. 107).

La furie des éléments a vite raison de cette assurance de la jeunesse et le mauvais temps (expression qui prend ici une connotation imprévue, toute positive) devient le lieu d'une renaissance:

Je n'existe déjà plus. Pourtant quelqu'un se lève et ce qu'il entreprend est impossible. [...] Alors le publiciste quinquagénaire monte sur le pont. Il sort complètement de son rôle. C'est lui qui devrait être terrorisé. C'est moi qui devrais le contraindre d'une poigne de fer, l'humilier, lui imposer ma volonté, le 
réduire à ma merci. [...] Véritablement dédoublé, je le regarde faire [...] et une sorte de tendresse me vient pour cet homme qui prend les coups à ma place, qui prend les coups depuis trente ans pendant que je peaufine ma belle âme (MT, p. 124125).

La troisième partie donne ensuite la parole à Monsieur Robert dans un plaidoyer qui défait une à une les certitudes de Bob et redore le blason de l'âge mûr. Au tour de Monsieur Robert de se payer la tête du jeunot qui n'aurait pas survécu à la tempête: "Vois-tu, Bob, [...] tu n'as jamais été brûlé par tes passions mais agité par des velléités. Tu es revenu de tout avant d'y être allé. $\mathrm{Tu}$ es moins malheureux que maussade. [...] Non, tu n'es pas Werther, mais Madame Bovary rêvant aux galeries Lafayette et croyant qu'elle aspire à l'infini " (MT, p. 140-141). L'homme mûr, justement à cause de cette maturité qui lui permet de voir mieux et plus loin, supplie Bob de ne pas disparaître pourtant, de continuer à lui insuffler ces inflexions de révolte dont il aura toujours besoin pour continuer à avancer: "Reste avec moi, Bob, ou plutôt reste avec nous. [...] Ta raideur, tes intransigeances me sont nécessaires et ta mauvaise conscience aussi qui me protège de la somnolence" (MT, p. 146-147). La conciliation est consommée, pour ainsi dire, et le nous, devenu un je enrichi de tous les aspects de l'expérience, est prêt à poursuivre la relation avec Anne.

Ainsi donc, chez Guimard, l'action détermine la structure du roman: les changements de voix narratives se découpent nettement pour bien marquer la conciliation des instances en présence. Tout est dit ("J'aurai grand besoin de toi", p. 141) dans une impudeur qui contraste singulièrement avec les procédés de La part des choses où les questions sont à peine posées et les réponses demeurent à deviner. Au moment de quitter Yves, qui reste sur le Moana pour poursuivre la production du film dans laquelle il s'est engagé, Marion, forte du retour sur elle-même et sur l'autre qu'elle a effectué pendant le voyage - et à travers le cahier Gallia — "s'enhardit à demander: "Les morts finissent par couler, non... - Oui, mon chéri, dit Yves. Heureusement." Elle ne savait pas quoi répondre quand Yves laissait échapper sa vérité" ( $P C$, p. 255). Ces bribes métaphoriques, allusion à Yang qui continuait de les hanter, signent la trêve entre les époux, une trêve qui se consomme en sous-entendus et à demi-mots, comme si les personnages eux-mêmes craignaient de tout gâcher par des explications inopportunes: "Ils se quittèrent en riant. Seule un 
peu de brume dans le regard de Marion l'empêcha de voir celle du regard d'Yves" ( $P C$, p. 259).

Dans ce roman aussi, cependant, les choix narratologiques s'inscrivent dans un processus symbolique: à l'avant-dernier chapitre du roman, en effet, s'opère une fusion entre la narration extérieure et le cahier Gallia, faisant basculer le texte du elle au je, comme pour signifier chez Marion la reprise définitive du contrôle de sa vie. Le je qui s'exprime désormais n'a plus besoin du confort de l'intimité diaristique pour se dire: il s'affiche en dehors du cahier Gallia, pour marquer la prise en charge d'un nouveau quotidien, cette fois débarrassé des rancunes et des amertumes.

Et comme pour souligner un retour - souhaité- à la "normale", le tout dernier chapitre redonnera la parole à une narratrice extérieure qui parle du couple en termes de elle et de lui, comme si les protagonistes s'étaient même désintéressés de leur propre rôle dans le roman, trop occupés à savourer leur existence en paix. Comme chaque matin, "ils" vont à la pêche, sur cette mer bretonne témoin de leurs crêtes et leurs creux conjugaux:

L'homme descend la prame à la lisière du flot. Les oiseaux ne se dérangent pas: ce n'est pas l'heure d'avoir peur; chacun est là parce qu'il doit y être. La femme se met aux avirons. Les bruits non plus ne sont pas réveillés, ils sont comme étouffés et ne font pas leur vrai bruit. [...] La femme étale une toile bleue sur le pont du bord où ils relèveront le tramail, sort le croc, la gaffe, l'écailloir. [...] Ils ne parlent pas. Pourquoi faire? Ils connaissent chaque geste à exécuter, chacun sait ce que fait l'autre à tout moment et ils savent qu'ils sont heureux (PC, p. 282283).

Deux romans, deux quêtes personnelles qui aboutissent à des formes différentes de sérénité. Dans les deux cas, la mer joue le rôle d'entremetteuse dans la mesure où elle constitue le pont entre une vie antérieure et une vie à venir, elle-même lieu de jonction avec un présent tourmenté qui appelle un coup de barre.

\section{Temps et généricité}

C'est à travers leur façon d'inscrire le temps dans la fiction, cependant, que les deux auteurs marqueront davantage leur différence sexuelle, inscrivant du même coup le profond écart qui sépare l'homme et la femme face au cours des choses. Ici, la fiction, si elle conserve bien sûr toute son importance, s'efface tout 
de même un peu au profit d'une réflexion élargie et plus résolument sociale qui met en relief l'idée de l'âge comme élément de mesure de l'individu.

Les deux romans peuvent en effet être lus comme autant de miroirs d'une même problématique, à savoir les difficultés de vieillir dans une société totalement tournée vers la jeunesse. Cependant, les auteurs adoptent des points de vue susceptibles de relever plutôt l'inégalité des sexes dans cette lutte contre le temps. À cet égard, la position de Groult se révèle beaucoup plus critique que celle de Guimard: l'auteure dénonce avec vigueur l'éternelle précarité de la femme face au temps qui, dès la quarantaine, voire la trentaine, commence à ressentir durement le choc des préjugés, l'injustice de la discrimination dont elle est victime, discrimination qui croît à mesure qu'elle s'éloigne "du type idéal d'humanité" ( $P C$, p. 78). "Le vieillissement, c'est le défaut numéro un, la faute inexpiable, et il nous faut "faire jeune" sous peine de mort quand on est une femme ", écrit Groult, dans un de ses nombreux éditoriaux sur la question ${ }^{7}$, et qui conclut ainsi: "Il reste aux femmes à conquérir le droit... de ne plus être jeunes ${ }^{8}$ ".

Chez Paul Guimard, la question du vieillissement et des effets du temps est abordée sous un angle en apparence plus universel; nous portons tous en nous, effectivement, un autre nous-mêmes plus jeune, dont il faut un jour ou l'autre divorcer pour accéder à la maturité. Nous pouvons dès lors plus facilement, hommes ou femmes, faire nôtres les remises en question de Monsieur Robert. Pourtant, l'aventure du personnage est, au-delà de la seule appartenance sexuelle, profondément masculine; les grands thèmes évoqués - le mirage de la jeunesse dont il faut réévaluer le fallacieux éclat, la force de l'expérience, etc. - s'appuient sur un fait romanesque incontournable qu'une lecture au féminin (ou même une simple lecture honnête de la temporalité) aura tôt fait de relever: Monsieur Robert, quinquagénaire, va poursuivre sa vie avec une jeune femme de trente ans avec qui il envisage d'avoir un enfant.

6 Benoîte Groult, La moitié de la terre, Paris, Alain Moreau, coll. "Pressepoche", 1981, p. 15.

7 Ces articles sont parus d'abord en tant qu'éditoriaux dans la revue F Magazine dont Benoite Groult était la rédactrice en chef.

8 Benoîte Groult, La moitié de la terre, op. cit., p. 17. 
Ce détail, qui n'en est pas un, cristallise en fait l'écart des déterminismes masculins et féminins devant le temps: outre la tolérance plus grande dont fait preuve la société à l'égard de la maturité masculine (on est vieux moins jeune quand on est homme), l'horloge biologique achève de maintenir la femme dans un état d'asservissement propre à commander les étapes de son existence ${ }^{9}$. Ainsi donc, on peut être tenté de refaire la lecture du Mauvais temps sous l'angle du féminin et mesurer cette quête de soi à l'aune des recommencements toujours possibles; cette nouvelle perception n'invalidera certes pas la première mais replacera la démarche du personnage dans le contexte d'un continuum plutôt que d'un ultimatum, comme ce sera le cas dans La part des choses.

Le roman de Groult expose en effet l'urgence et la difficulté pour la femme de faire du temps un allié et non plus un ennemi implacable. Son personnage, à l'encontre de Monsieur Robert, voit "arriver l'heure des moindres chances et des "plus jamais" 10 ". Marion est à la croisée des chemins, à un moment plus déterminant que celui vécu par son homologue masculin du Mauvais temps. Les personnages qui gravitent autour de Marion lui serviront en quelque sorte de repoussoir, lui fournissant des balises pour évaluer sa propre position. À peu près tous de la même génération et présentés d'abord par leur âge, ils résument des situations somme toute banales dans leur diversité et incarnent par leur comportement des exemples de vie (à suivre et à ne pas suivre) qui apporteront des réponses à Marion.

Chacun de ses compagnons de voyage privilégie "une certaine manière de vivre le temps, de poser son existence dans, en face de, contre le temps qui surgit en lui ${ }^{11}$ ". Ainsi Iris, 50 ans, prototype de la "femme qui a déjà été belle", ne voit dans l'avenir que la perte des privilèges reliés à la beauté. Son obsession de la vieillesse, qui lui fait rater l'instant présent, détournera d'elle son mari, Alex, 52 ans, qui tombera amoureux de Betty, 26 ans, la scripte-girl; Jacques, victime d'un infarctus à 43 ans, a retrouvé

9 Que les femmes décident actuellement d'avoir leurs enfants à un âge plus avancé ne change rien à la question : il existera toujours un moment où elles "ne pourront plus" et elles doivent faire leur choix de vie en conséquence.

10 Michèle Thiriet et Suzanne Képès, Femmes à 50 ans, Paris, Éditions du Seuil, 1981, p. 102.

11 Pierre Burgelin, op. cit., p. 10. 
durant sa convalescence le plaisir de vivre mais découvre en même temps qu'il ne peut plus supporter sa femme Patricia, 42 ans, ménagère accomplie, qu'il abandonne avec ses cinq enfants en choisissant de demeurer à Tahiti. Au centre de ces couples qui se défont, Marion et Yves survivront à tous ces naufrages en harmonisant, précisément, leur façon d'appréhender le temps.

Car Marion - représentative en cela d'une certaine expérience féminine de la contrainte temporelle - est une personne de la $d u$ rée, comme on dit de quelqu'un qu'il est du matin. Toute sa vie s'inscrit dans la durée et les cycles qui la nourrissent: elle déteste les départs, les ruptures, préférant de loin les rituels ordinaires, le prévisible déroulement, mais pour elle jamais banal, du quotidien. Les bruits familiers, les odeurs retrouvées, les formules convenues que l'on échange avec le facteur après une absence, bref les menus faits qui composent une existence ancrent profondément Marion dans son temps. Yves, au contraire, ne vit que dans l'instant: moment d'une nouvelle conquête, fuite vers de nouveaux horizons, refus de tout engagement dont il ne peut prévoir l'issue.

Les deux personnages vivent le temps de façons diamétralement opposées; cette profonde - et en apparence irréconciliable- divergence, cause partielle de la distance affective qui les sépare, se trouve métaphorisée à travers tout le roman: dans les dialogues, par des phrases anodines telles que " $C e$ qu'il y a de beau dans la navigation, c'est de débarquer, tu ne trouves pas?" dit Marion à Yves qui pensait justement le contraire" ( $P C$, p. 106); dans les réflexions du cahier Gallia: "Nous avons rarement des moments de vraie intimité. Or, tout est une question de moment avec lui: il faudrait lui sauter dessus comme un tigre..." $(P C$, p. 192); dans la narration, qui donne accès aux pensées d'Yves: "[Il] ne réclamait plus qu'une seule chose: l'indifférence. Il avait besoin de camarades qui ne soient pas des amis, de pays qui ne soit pas le sien, de vieilles dames qui ne soient pas sa mère, de village qui ne ressemblent pas à Kerviniec, de problèmes qui ne le concernent pas" ( $P C$, p. 146).

Le long voyage entrepris par le Moana sera l'occasion pour les deux époux d'amalgamer leur façon de vivre le temps, en en prenant pleinement conscience d'abord et en la mesurant à celle de l'autre ensuite. Marion apprendra à saisir l'instant au vol; elle découvrira "l'égoïsme, ce vice indispensable à la santé" $(P C$, p. 246) et s'autorisera symboliquement une aventure sans lendemain avec un Américain. Elle écrit alors ces lignes dans son ca- 
hier Gallia, en s'adressant à Yves: "Je vais faire l'amour comme une Tahitienne, tu vois, c'est contagieux; ou plus simplement comme un homme. Mais je ne te le dirai pas. Tu ne reviens que dans deux mois, j'aurai oublié" ( $P C$, p. 266). Yves pour sa part se rend compte, non sans une certaine confusion, qu'il est lié à Marion par des "sentiments hélas profonds, hélas sincères" $(P C$, p. 121). La narratrice exprime alors pour lui l'effet d'une durée qu'il avait jusque là soigneusement évité d'assumer :

Ces êtres-là ne se rendent pas compte [...] qu'en vivant un peu longuement avec la même femme, elle entre peu à peu dans leur paysage le plus intime, dans leurs fibres, dans leur passé, et qu'elle devient inséparable d'eux-mêmes sans qu'ils s'en aperçoivent. Au bout d'un nombre d'années suffisant, ils sont organiquement incapables de se défaire d'elles sans se détruire. Elle est tissée à leur vie, comme une laine d'une autre couleur qu'on a tricotée avec la vraie sans trop y prendre garde et qu'on ne peut plus séparer sous peine de démolir tout l'ouvrage. Il y avait une couleur Yang sur une portion de l'ouvrage d'Yves. Puis le fil avait manqué. Celui de Marion courait avant, pendant, après, volubilis interminable qu'Yves continuait à tricoter [...] (PC, p. 254).

Temps de la réconciliation et réconciliation des temps, la croisière sur le Moana est, en fait, un nouveau départ pour les deux époux alors que chez Guimard, la navigation oblige le marin à se concentrer sur lui-même pour opérer la fusion entre les deux moi qui l'habitent. Ces deux démarches apparentées mais bien différentes reposent toutefois sur une ironie partagée, élément qui, d'une façon diffuse mais tout de même palpable, scelle le pacte de parenté entre les deux écritures.

\section{Ironie, lucidité et cruauté}

Cette ironie prendra pour principal appui, dans une cohérence que les romans ne démentent jamais, le temps, ses effets, ses conséquences: elle sera tour à tour indulgence, satirique, impitoyable, au gré de l'intention des ironistes qui s'assurent ainsi une distance nécessaire par rapport à des situations qui, autrement, confineraient au tragique. Encore ici, cependant, se pressent l'écart entre les perspectives masculine et féminine de soi et du monde.

D'un point de vue narratologique d'abord, l'ironie chez Guimard s'affirme par le biais d'un je polymorphe, comme on l'a 
134

vu, mais dont la double nature s'estompe au fil du roman. Monsieur Robert et Bob sont en effet très critiques l'un envers l'autre, prompts à pointer du doigt leur déchéance respective. Pourtant, l'ironie émane surtout de Bob, dans la première partie, pour se faire beaucoup plus rare dans la troisième, au moment où Monsieur Robert prend la relève pour les sauver tous deux du naufrage. Paradoxalement, l'ironie, en principe l'arme du fort, n'est utilisée - par Bob - que pour tenter de juguler, précisément, la puissance de Monsieur Robert qui s'imposera à la fin.

Bob, manifestement, redoute de disparaître pour de bon et multiplie les attaques, sans doute pour attirer la sympathie d'un lecteur qui, effectivement, rira avec lui de la décadence de son vieil homologue:

Je dois veiller sur [Anne]. L'autre supporte mal d'être tenu à l'écart. Anne l'intéresse pour des raisons qui n'ont rien à voir avec l'art religieux. Monsieur Robert a fait l'inventaire du corps d'Anne et souhaite s'en régaler à la première occasion. Il ne s'arrête pas sur ce qui, dans ce corps, atteint à la perfection. Par exemple la ligne souple que composent les contours du visage, du cou très long et de l'attache des épaules, cette flexibilité qui fascinait Modigliani et que l'on retrouve chez les hautes femmes du Nil. Monsieur Robert, lui, s'attarde sur la viande. Et d'abord sur le cul un peu lourd, duquel il se promet de bons moments. A défaut d'oser les gestes que je parviens encore à lui interdire, il fait la roue. Il sollicite l'attention. Il charge mon regard d'une langueur ridicule. Il lisse du bouts des doigts les cheveux follets du dessus de l'oreille avec le geste stéréotypé des mâles latins en représentation. [...] Jusqu'ici, les tentatives de séduction de Monsieur Robert ont fait long feu. C'est à moi, Bob, qu'Anne parle, c'est moi qu'elle regarde et qu'elle entend. Relégué au vestiaire, en compagnie du manteau de cashmere dont il tire vanité, l'Autre se morfond à rêver de fesses tandis que nous parlons de cette Nativité de La Tour dont je ne jurerais pas qu'elle soit accrochée au musée de Nantes [...] (MT, p. 21-22).

Ce passage met à jour la stratégie du roman: on y sent le mépris du jeune pour le "vieux" qu'il est en train de devenir mais on remarque, dans la chute de la phrase, dans ce je ne jurerais pas, l'incertitude d'une culture usurpée, née de l'expérience qu'on a encore à acquérir. Le combat que livre Bob à son alter ego (ou peut-être devrait-on dire son ego altéré) ressemble par moment à une lutte rhétorique où la prouesse de l'écriture l'emporte haut la main sur le déroulement romanesque. Les anecdotes prennent 
leur sens dans la recherche stylistique qui fait même perdre de vue, à certains endroits, l'importance des enjeux. "Il y a longtemps que je ferme à minuit", soupire ainsi Bob, devenu Monsieur Robert ( $M T$, p. 47), dans une métaphore qui déplore l'énergie perdue d'une jeunesse qui, contrairement à ce que l'on croit à vingt ans, n'est pas éternelle...

Presque entièrement orchestrée autour du héros, l'ironie ne prend que rarement à partie le reste de l'univers, si ce n'est pour relancer sa critique de Monsieur Robert qui, par ailleurs, avec la magnanimité de celui qui remporte la victoire finale, refuse de répondre sur le même ton: "Il me semble que l'essentiel de tes reproches s'adresse autant au vieillard futur qu'au jeune homme ancien. Ce que nous sommes aujourd'hui, tu l'escamotes. [...] Ta difficulté d'être tient à ton insurrection permanente contre ce dénouement et je voudrais conjurer tes erreurs d'outre-tombe qui nous empoisonnent l'existence" (MT, p. 142).

L'être serein et en pleine possession de ses moyens laisse l'ironie aux autres, semble suggérer Guimard ${ }^{12}$. Cependant, l'auto-ironie omniprésente dans les deux premières parties - un avatar de l'auto-dérision, devrais-je dire, puisqu'elle ne s'adresse qu'à une moitié du je en présence - tend aussi à laisser croire que cette critique est nécessaire pour aboutir à la paix intérieure ; c'est le "Cap Horn" dont parle Benoîte Groult, c'est-à-dire le moment où l'on est aspiré "contre son gré dans l'affreux défilé où il faudra subir l'inacceptable, admettre des réalités qui vous tuent, se résigner à tout ce qu'on s'était tant juré de refuser à vingt ans " $(P C$, p. 52). La résistance devant l'inévitable passerait donc par l'ironie...

C'est aussi l'opinion de Groult qui pose sa narratrice en critique impitoyable des compatriotes de Marion et fait de son héroïne une observatrice dont les pointes ironiques acérées accusent une absence totale de complaisance. D'ailleurs, dans ce roman, contrairement à celui de Guimard qui opère une fusion entre les deux moitiés du je par le biais d'un renoncement à l'ironie, l'amalgame des instances en présence se fera dans l'ironie, entre la voix de Marion du cahier Gallia et la voix de l'ironiste

12 Dans ce roman, à tout le moins, car dans les autres ouvres de Guimard, le narrateur-ironiste s'en donne à cour joie: ce sont les choix narratologiques bien particuliers du Mauvais temps qui appellent ce constat. 
qui impose, ni plus ni moins, ses inflexions à la narratrice, comme si Groult elle-même voulait signifier sa présence au lecteur en commentant, dans un troisième palier de narration, ce qu'elle fait dire à sa narratrice. Un exemple de cette intrusion de l'auteure: "Hautains et distants comme leurs chameaux, [...] des nomades traversaient les faubourgs au rythme saccadé de leurs bêtes, les plus sinistres animaux de la création, et quelqu'un fit remarquer que les hommes étaient pleins de noblesse et qu'ils drapaient leurs turbans avec un chic fou. C'est généralement ce qu'on dit des nomades" (PC, p. 110-111. Je souligne).

Ce ton, récurrent dans tout le roman, rejoint en effet la distance que Marion privilégie pour analyser les événements du voyage et pour faire le bilan de sa vie en général. Qu'on en juge par la phrase suivante, dont on ne sait plus si elle émane de la conscience de Marion, de la narratrice ou de l'ironiste: à l'aube du grand départ, "l'heure de décevoir les autres et elle-même avait sonné" ( $P C$, p. 23). Par l'ironie s'effectue subtilement une osmose entre Marion et Groult qui tend à effacer l'écart narratologique pour affirmer le rôle non plus d'une instance abstraite mais de l'auteure elle-même dans le processus ironique ${ }^{13}$ et pour réactualiser la question de la généricité.

Le regard que porte Marion sur sa jeunesse, par exemple, se révélera radicalement différent de la nostalgie que nourrit Bob pour ses "belles" années, et sera marqué de la différence sexuelle dans la mesure où, comme en fait foi l'extrait suivant, le texte résume la condition de toute une génération de femmes et souligne l'absence de la réciproque masculine:

On me poussa affectueusement vers la Sorbonne, un ou deux certificats de lettres "en attendant" n'ayant jamais empêché une jeune fille de se marier. Malheureusement l'attente s'était transformée en licence classique puis en diplômes d'études supérieures avant qu'un candidat offrant des garanties suffisantes n'eût été agréé par le tribunal familial. Il était temps: le spectre de l'agrégation qui fait les vieilles filles à lunettes hantait déjà mes parents.

Le souvenir d'avoir été un bestiau à la foire, sur lequel les acheteurs ne s'étaient pas précipités assez vite malgré les bichonna-

13 On n'a pas cette même impression de nivellement auteur-narrateur dans Le mauvais temps, encore une fois à cause des choix narratologiques. 
ges de ses soigneurs, me laissa pour des années une humilité hargneuse à l'égard des hommes $(P C, \mathrm{p} .81)$.

Voilà tracé, par une auto-ironie assez cruelle, l'itinéraire féminin usuel avant la montée du féminisme; on y sent les accents militants (et biographiques!) de Groult; toutefois, à l'encontre des auteures québécoises, elle exercera son ironie aussi - et avec une égale férocité- sur ses pairs ${ }^{14}$. Si les hommes passent allègrement à la moulinette de l'ironie de la narratrice, celle-ci, en effet, ne ménagera aucunement les voyageuses, disant notamment, de Patricia, et des nombreuses maternités dont elle s'enorgueillit: "Ce sont des choses plus faciles à faire qu'à ne pas faire, bien que les félicitations des amis et de la société tendent à faire croire aux parents qu'ils viennent de réussir un exploit. Félicite-t-on une machine à cachous de débiter ses cachous dans le métro?" ( $P C$, p. 37).

Cette causticité, qui commente la traversée, suit un processus légèrement différent de celui de Guimard. Rentrée de voyage, en paix avec elle-même, Marion - et la narratrice qui la guide n'abandonnera pas totalement l'ironie pour laisser parler la voix de la sérénité. Plus joyeuse, toutefois, la moquerie servira plutôt à insister sur le fait que Marion est revenue transformée de son périple pour reprendre sa place dans un monde qui, lui, est resté le même, pour son plus grand bonheur. Le passage suivant agit comme métaphore de l'assurance qu'a prise l'héroïne au cours des derniers mois et du plaisir mutin qu'elle éprouve à s'amarrer de nouveau au quotidien:

C'est l'heure où les touristes ouvrent à leur tour les volets de leurs villas et s'exclament: "Zut, il pleut!" [...] Courageusement parce qu'en Bretagne les vacanciers ont le sens du devoir et le souci de profiter de l'iode, ils descendront [...] chargés du matériel toujours plus perfectionné du plagiste, peignoirs-cabines, seaux, pelles, [...] le pliant de Mémé qui trouve la terre basse, l'ouvrage de maman qui tricote déjà pour l'hiver, le transistor pour papa qui s'ennuie toujours sur le sable et qui ne veut pas manquer son match [...]. Sur la route de la côte les plagistes croisent l'homme et la femme qui remontent en se dandinant un peu, avec le panier d'où dépasse le museau de la sole de

14 De leur côté, les auteures québécoises, à la même période, répugnaient à faire de l'ironie au détriment des femmes. Voir mon ouvrage: Le Carquois de velours. L'ironie au féminin dans la littérature québécoise (1960-1980), Montréal, l'Hexagone, 1998. 
138

trois livres, la face blanche sur le dessus. Ils ont tout calculé pour qu'un petit Parisien s'approche et crie: "Papa, viens voir cette bête énorme, qu'est-ce que c'est? " pour qu'un père héroïquement vêtu d'un short, comme tous ceux qui aiment donner des leçons de choses, s'approche et dise d'un ton docte: "Ça, tu vois, c'est une limande..." et répondre d'un ton neutre: "C'est une sole, monsieur" puis s'éloigner, modeste, en traînant ses bottes (PC, p. 285-286).

Ainsi donc, Groult et Guimard se répondent par romans interposés. Ils proposent des visages différents d'une même réalité, ressemblance qu'ils aiguisent encore davantage par le choix de décors communs - la Bretagne, entre autres-, de thématiques apparentées ou de préoccupations convergentes. Leurs voix se fusionnent tout en évitant "l'accord total qu'on n'entend plus à la longue tant il est parfait" ( $P C$, p. 254). Mais les deux romans demeurent marqués par l'appartenance sexuelle de leurs auteurs: même à travers la fiction - à travers la fiction du temps surtout - se lit la profonde différence des expériences féminine et masculine de l'existence. 